\title{
A predictive nomogram for mortality of cancer patients with invasive candidiasis: a 10-year study in a cancer center of North China
}

\author{
Ding $\mathrm{Li}^{1^{*}}$, Tianjiao Li ${ }^{2}$, Changsen Bai ${ }^{1}$, Qing Zhang ${ }^{1}$, Zheng $\mathrm{Li}^{1}$ and Xichuan $\mathrm{Li}^{3^{*}}$
}

\begin{abstract}
Background: Invasive candidiasis is the most common fungal disease among hospitalized patients and continues to be a major cause of mortality. Risk factors for mortality have been studied previously but rarely developed into a predictive nomogram, especially for cancer patients. We constructed a nomogram for mortality prediction based on a retrospective review of 10 years of data for cancer patients with invasive candidiasis.

Methods: Clinical data for cancer patients with invasive candidiasis during the period of 2010-2019 were studied; the cases were randomly divided into training and validation cohorts. Variables in the training cohort were subjected to a predictive nomogram based on multivariate logistic regression analysis and a stepwise algorithm. We assessed the performance of the nomogram through the area under the receiver operating characteristic (ROC) curve (AUC) and decision curve analysis (DCA) in both the training and validation cohorts.

Results: A total of 207 cases of invasive candidiasis were examined, and the crude 30-day mortality was 28.0\%. Candida albicans (48.3\%) was the predominant species responsible for infection, followed by the Candida glabrata complex (24.2\%) and Candida tropicalis (10.1\%). The training and validation cohorts contained 147 and 60 cases, respectively. The predictive nomogram consisted of bloodstream infections, intensive care unit (ICU) admitted $>3$ days, no prior surgery, metastasis and no source control. The AUCs of the training and validation cohorts were 0.895 (95\% confidence interval [Cl], 0.846-0.945) and 0.862 (95\% Cl, 0.770-0.955), respectively. The net benefit of the model performed better than "treatment for all" in DCA and was also better for opting low-risk patients out of treatment than "treatment for none" in opt-out DCA.

(Continued on next page)
\end{abstract}

\footnotetext{
*Correspondence: lidingly@126.com; xichuanli@tmu.edu.cn

'Department of Clinical Laboratory, Tianjin Medical University Cancer Institute and Hospital, National Clinical Research Center for Cancer, Key Laboratory of Cancer Prevention and Therapy, Tianjin's Clinical Research Center for Cancer, Huanhu West Road, Hexi District, Tianjin 300060, China ${ }^{3}$ Tianjin Key Laboratory of Animal and Plant Resistance, College of Life Sciences, Tianjin Normal University, Binshuixi Road, Tianjin 300387, Xiqing District, China

Full list of author information is available at the end of the article
}

(C) The Author(s). 2021 Open Access This article is licensed under a Creative Commons Attribution 4.0 International License, which permits use, sharing, adaptation, distribution and reproduction in any medium or format, as long as you give appropriate credit to the original author(s) and the source, provide a link to the Creative Commons licence, and indicate if changes were made. The images or other third party material in this article are included in the article's Creative Commons licence, unless indicated otherwise in a credit line to the material. If material is not included in the article's Creative Commons licence and your intended use is not permitted by statutory regulation or exceeds the permitted use, you will need to obtain permission directly from the copyright holder. To view a copy of this licence, visit http://creativecommons.org/licenses/by/4.0/ The Creative Commons Public Domain Dedication waiver (http://creativecommons.org/publicdomain/zero/1.0/) applies to the data made available in this article, unless otherwise stated in a credit line to the data. 
(Continued from previous page)

Conclusion: Cancer patients with invasive candidiasis exhibit high crude mortality. The predictive nomogram established in this study can provide a probability of mortality for a given patient, which will be beneficial for therapeutic strategies and outcome improvement.

Keywords: Invasive candidiasis, Mortality, Predictive nomogram, Candida, 30-day death

\section{Background}

Invasive candidiasis refers to bloodstream (that is, candidemia) and deep-seated infections by Candida spp., a common opportunistic pathogenic fungus that is commensal on the human body surface, including the skin, oral cavity, intestines and vagina [1]. Candida ranks as the fourth most prevalent nosocomial pathogen of bloodstream infection in the United States and seventh to tenth in population-based studies, with the C. albicans, C. tropicalis, C. glabrata and C. parapsilosis complex comprising the vast majority causal agents of the disease [2,3]. Although the diagnostic tools and management strategies of invasive candidiasis have been improved, it is still a deadly infection found all over the world, and the mortality can reach $40 \%$ and even higher among patients with malignancy or those who are critically ill $[4,5]$.

Invasive candidiasis represents a major challenge among healthcare-related infections due to its difficult diagnostic and therapeutic management [6]. Therefore, a better understanding of the underlying risk factors for the development of infection and mortality is of high clinical importance. A number of studies have found that mortality independently increases with elderly age, renal failure, malignant diseases, central venous catheterization $(\mathrm{CVC})$, steroid therapy, admission to an intensive care unit (ICU), use of total parenteral nutrition (TPN), low lymphocyte count, gastrointestinal source of candidemia, or previous exposure to antibiotics $[1,7,8]$. However, these predictors contribute little to obtaining a better prognosis and always vary among populations. Thus, we speculated that a model combining different risk factors (cumulative number) might provide a better prediction for the outcome of invasive candidiasis than a single factor.

A large proportion of cancer patients with both hematologic and solid malignancies are susceptible to invasive candidiasis and have poor outcomes [9]. In other words, invasive candidiasis greatly reduces the survival rate of cancer patients. To our knowledge, research on prognosis prediction for invasive candidiasis among cancer patients is limited, and predictive models are rarely studied. In the present study, we constructed a predictive nomogram based on prognosis predictors of cancer patients with invasive candidiasis and showed that this nomogram has a good mortality prediction ability in both training and validation cohorts. Thus, we believe that the nomogram constructed in the study will provide a better prognosis prediction than a single predictor and will benefit treatment strategies (such as strengthening source control and intravenous therapy) and increase the survival rate among cancer patients with invasive candidiasis.

\section{Methods \\ Study design}

This was a retrospective observational study, and data were collected from Tianjin Medical University Cancer Institute and Hospital, among the top cancer institutes in China. To construct the predictive nomogram for 30day death of invasive candidiasis among cancer patients, we first randomly divided patient data into training and validation cohorts. The predictive nomogram model was constructed according to the data in the training cohort, and the data in the validation cohort were verified by using the same regression equations that were constructed for the training cohort. The ability of this predictive nomogram was assessed by using a calibration plot of the area under the receiver operating characteristic (ROC) curve (AUC), and the clinical usefulness was examined via net benefit by using decision curve analysis (DCA).

This study obtained permission from the Bioethics Committee of Tianjin Medical University Cancer Institute and Hospital and participants (consent to participate was obtained from all participants or their parents or legal guardians for participants under 16 years old) to review patient records and use the data.

\section{Data collection}

Cancer patients who were hospitalized during the period from 2010 to 2019 and had been diagnosed with invasive candidiasis were eligible for inclusion. Patients without a pathologic diagnosis of cancer, with polymicrobial or multiple-site infections, who had recurrent invasive candidiasis (occurring more than 30 days after the initial episode), or who died earlier than the initial positive culture were excluded; a flow chart of patients excluded for each criterion is shown in Fig. 1. Candida species were evaluated. Clinical data collected from medical reports included age, gender, length of hospital stay (LOS) to the initial positive culture, types of cancer, site of 


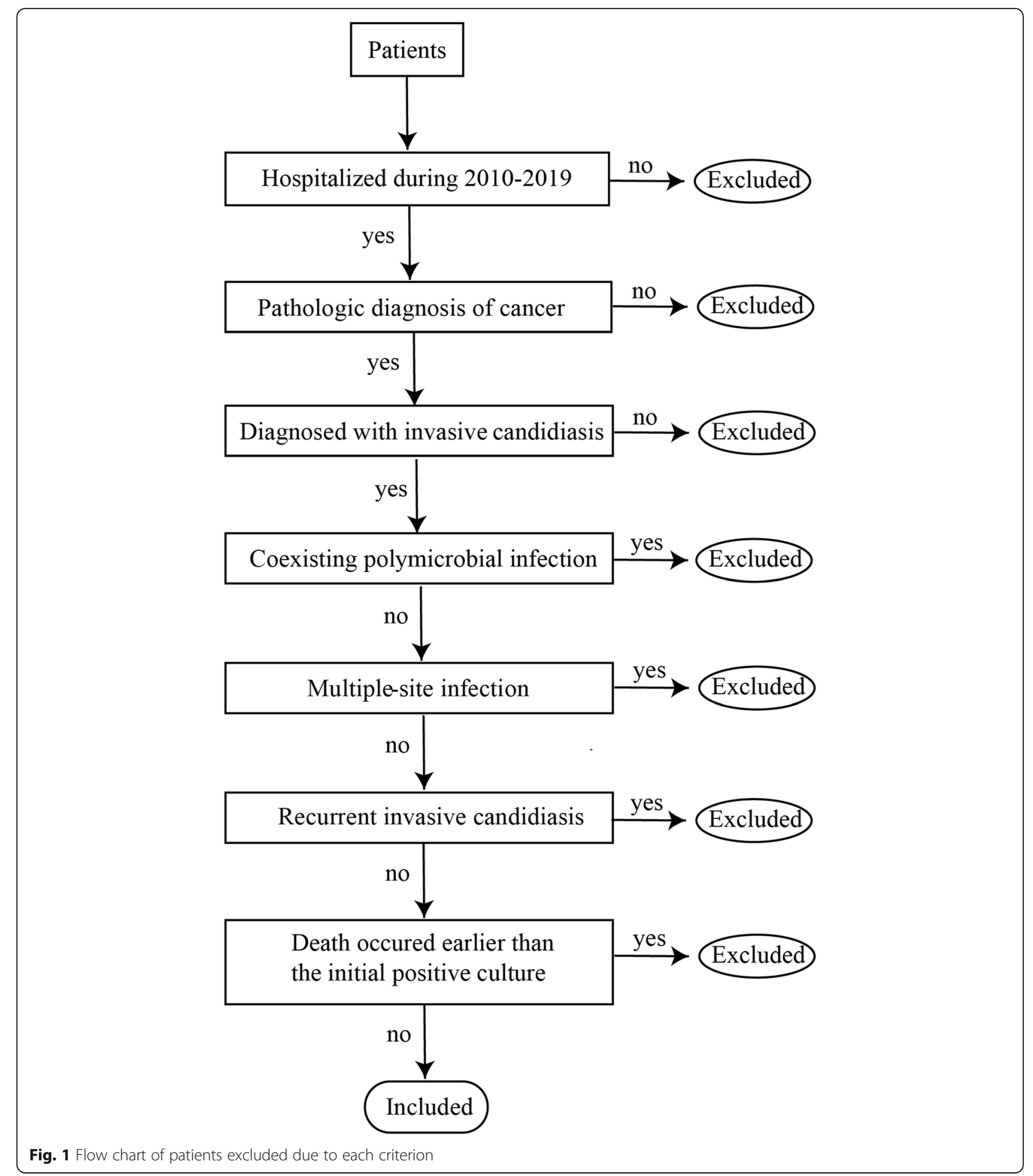

infection, tumor metastasis, predisposing factors (chemotherapy, neutropenia, surgery, prior antibiotic use, CVC retention $>7$ days, urinary catheter retention $>2$ days, nasogastric tube retention $>3$ days, ICU admitted $>3$ days, TPN administration $>5$ days) present at or within the last 30 days prior to the initial positive culture, comorbidities (diabetes mellitus, live dysfunction, kidney dysfunction, respiratory dysfunction, cardiovascular dysfunction) present within the last 30 days prior to the initial positive culture or during the progression of the invasive candidiasis, and antifungal treatment (fluconazole prophylaxis before confirmation of invasive 
candidiasis, timely antifungal treatment and source control after diagnosis). All data and outcomes were collected on day 30 after the date of the initial positive culture or death if this occurred earlier. There were no missing values in this study.

\section{Definitions}

A case diagnosed as invasive candidiasis was defined based on isolation of Candida spp. from bloodstream or other normally sterile sites, and isolation of Candida $s p p$. from bloodstream was also called bloodstream infection $[5,10]$. Polymicrobial infection referred to infection by more than one pathogen at the same time. Site of infection referred to the site from which Candida was isolated, including the bloodstream, intra-abdominal, intrathoracic and intrapelvic cavities. All deep-seated samples were obtained via paracentesis or surgery. Types of cancer were differentially diagnosed by pathological examination. Cancers occurring in the stomach, duodenum, colon or rectum were referred to as gastrointestinal cancer; cancers occurring in the bile duct, liver or pancreas were referred to as hepatic-pancreatic cancer. Neutropenia was defined as an absolute neutrophil count of $<1.5 \times 10^{9} / \mathrm{L}$. Comorbidities were determined by the attending physician as follows: 1) insulin dependent diabetes; 2) decompensated cirrhosis; 3) episodes of hepatic failure; 4) hepatic encephalopathy; 5) dialysis dependent renal disease; 6) respiratory dysfunction including dyspnea on exertion, chronic respiratory hypoxia, or pulmonary hypertension; 7) cardiovascular dysfunction was defined as New York Heart Association grades III to IV $[11,12]$. Crude mortality referred to the rate of death within 30 days after the initial positive culture. Predictors were specified with worse outcomes in the study.

\section{Candida spp. identification}

Blood samples $(8-10 \mathrm{ml})$ were collected and autocultured using a BACTEC 9050, 9120 or FX (Becton-Dickinson, Franklin Lakes, NJ, USA) for 5 days; positive samples were subcultured on blood agar (JinZhangKeJi, Tianjin, China) at $35^{\circ} \mathrm{C}$ for $24-48 \mathrm{~h}$. Other sterile sourced samples were inoculated on agar with $5 \%$ sheep blood (JinZhangKeJi, Tianjin, China) for $24-48 \mathrm{~h}$ at $35^{\circ} \mathrm{C}$. candida spp. were preliminarily identified by Gram staining and then isolated using Sabourauds agar (JinZhangKeJi, Tianjin, China). Species identification was performed with a VITEK-2 Compact or VITEK-2 (bioMérieux AS, Marcy I'Etoile, France) by using purified yeast colonies.

\section{Statistical analysis}

Univariate analysis of categorical variables was performed using Fisher's exact test with SPSS 20.0 software
(SPSS Inc., Chicago, IL, USA), and all tests were 2-tailed. For dividing the data randomly, multivariable logistic regression, stepwise algorithm, nomogram construct, DCA, calibration and ROC analysis were performed with $\mathrm{R}$ version 4.0.2 or 3.6.1 ( $\mathrm{R}$ Core Team, Vienna, Austria). The significance level was set at $p<0.05$.

To screen predictors in the training cohort, Fisher's exact test was first performed. Then, clinical variables with $p<0.2$ were used to construct a predictive model by multivariable logistic regression analysis, and the best model was selected via a stepwise algorithm based on the minimal Akaike's information criterion (AIC). The variables in the predictive model we chose were applied to plot the predictive nomogram. The nomogram performance was composed of calibration and discrimination. Calibration was computed by comparing the predicted probability of 30-day death versus the actual probability of 30-day death in all patients, again using 1000 bootstrap resamples to reduce overfit bias, which would overstate the accuracy of the nomogram. Discrimination was quantified with the concordance index (Cindex). We evaluated the predictive ability of the nomogram via ROC analysis in the training cohort and verified it in the validation cohort. For clinical usefulness, net benefit was examined via DCA individually in the training and validation cohorts.

\section{Results}

\section{Distribution of Candida spp.}

In total, 207 cases of invasive candidiasis were examined in the current investigation, and 207 strains of Candida were isolated. C. albicans was the predominant species ( $n=100,48.3 \%$ ), followed by the C. glabrata complex $(n=50,24.2 \%), C$. tropicalis $(n=21,10.1 \%)$, the C. parapsilosis complex $(n=15,7.2 \%), C$. lusitaniae $(n=9$, 4.3\%), C. ciferrii $(\mathrm{n}=5,2.9 \%)$, C. famata $(\mathrm{n}=5,2.4 \%)$ and C. krusei ( $\mathrm{n}=2,0.1 \%)$.

\section{Clinical characteristics of patients and construction of a predictive nomogram}

For the 207 cases, the median age was 63 years old (range from 1 to 88 years old), and the median LOS was 23 days (range from 3 to 105 days). A total of 58 patients died within 1 mon during the study period, leading to a crude mortality of $28.0 \%$. The 207 cases were randomly split into two cohorts: a training cohort including 147 cases with $28.6 \%$ crude mortality and a validation cohort including 60 cases with $26.7 \%$ crude mortality. For the training cohort, the median age was 63 years old (range from 2 to 88 years old), and the median LOS was 24 days (range from 6 to 76 days). For the validation cohort, the median age was 61 years old (range from 1 to 86 years old), and the median LOS was 22 days (range from 3 to 105 days). None of the patients experienced organ 
infections due to Candida spp., and none of patients with bloodstream infections from deep seated infections during the study period. The other detailed clinical characteristics and results of univariate analysis are shown in Table 1.

To select the predictive model and plot the nomogram, we chose values of LOS $>=30$ days, no prior surgery, metastasis, ICU admitted > 3 days, no source control, bloodstream infection, abdominal cavity infection, and C. glabrata complex as candidate predictors of which $p<0.2$ in univariate analysis of the training cohort into multiple logistic regression and a stepwise algorithm. Then, we selected a predictive model containing predictors of bloodstream infection, ICU admitted $>3$ days, no prior surgery, metastasis and no source control based on a minimal AIC (Table 2), and a nomogram using these predictors as weights was built (Fig. 2).

\section{ROC analysis}

The predictive nomogram generates an individual numerical probability of 30-day death among cancer patients with invasive candidiasis, and the performance of the nomogram was evaluated by ROC analysis. Figure 3 shows the ROC curves for the training and validation cohorts. The AUCs of the training and validation cohorts were 0.895 (95\% confidence interval [CI], 0.846$0.945)$ and 0.862 (95\% CI, 0.770-0.955), respectively. In the training cohort, the cutoff value of the predictive score at the optimum point was 18.2 , the specificity was $67.7 \%$, and the sensitivity was $95.2 \%$. For the validation cohort, the specificity was $61.4 \%$, and the sensitivity was $93.8 \%$ when the cutoff value was set at 18.2 .

\section{Decision curve analysis}

DCA for the predictive nomogram model in the training and validation cohorts is shown in Fig. 4a and b, respectively. Based on the curves, the predictive nomogram actually benefits for treating high-risk patients, as indicated by the solid black line above the "treatment for all" line (which was approximately $>0.15$ of threshold probability for both cohorts). Opt-out DCA for the predictive nomogram in the training and validation cohorts is shown in Fig. 4c and d, respectively. The predictive nomogram showed a better performance for opting lowrisk patients out of treatment, as indicated by the solid black line above the "treatment for none" line (which was approximately $<0.8$ and $<0.7$ of threshold probability in the training and validation cohorts, respectively).

\section{Discussion}

In the whole cohort during the study period, the infections were mostly caused by $C$. albicans, followed by the C. glabrata complex, C. tropicalis, C. parapsilosis complex, C. lusitaniae and others. The crude 30-day mortality was $28.0 \%$, which is comparable to other studies (23.1-35\%) [13-15]. Risk factors for death from invasive candidiasis have been studied in recent decades and include older age, hematological malignancy, chronic organ dysfunction, and in the ICU at the time of diagnosis [8]. However, it is difficult to use these risk factors to predict prognosis accurately because we little is known about the weights of these factors in the outcome. A nomogram could solve this problem, and it is commonly used to estimate prognosis in medicine [16]. The userfriendly digital interfaces provide rapid computation for transforming complex clinical parameters into scores, making it easy to understand accurate prognoses and aiding in clinical decision making [17]. In the current study, we established a predictive nomogram (predictor variables were bloodstream infection, ICU admitted $>3$ days, no prior surgery, metastasis and no source control) for the prognosis estimation of invasive candidiasis among cancer patients.

ROC analysis is traditionally used to evaluate the performance of a model [18]. Based on the values of AUC, the predictive model constructed in this study has a good ability for prognosis prediction (the AUC was 0.895 in the training cohort and 0.862 in the validation cohort). However, an AUC alone is not sufficient to show that a model would improve decision-making. Vickers and Elkin introduced DCA to estimate the clinical utility of models firstly, and this approach has been widely used for evaluating predictive models and diagnostic tests in recent years [19]. Indeed, DCA can assess the net benefit of nomogram-assisted decisions at different threshold probabilities compared to the net benefit of decisions made with the assumption that the outcome of interest occurs for either all patients or no patient. Another common formulation of DCA is based on an opt-out framework (referred to as opt-out DCA), which displays the population net benefit of the risk model in comparison to the reference policy of treating no patients and opting low-risk patients out of treatment [2022]. In the training and validation cohorts of the current study, DCA showed that the predictive nomogram performs better than "treatment for all"; opt-out DCA indicated that the model benefits a decision to not undergo some intervention when treat none is the standard. Overall, the current predictive nomogram exhibited good performance regarding outcome prediction and can be applied for some opt-out strategies for invasive candidiasis among cancer patients.

The predictive nomogram constructed in the present study was based on a cancer population, which has been found to be much more susceptible to invasive candidiasis and shows high mortality. There were five predictors in this model, as follows: bloodstream infection, ICU 
Table 1 Clinical characteristics of patients with invasive candidiasis for crude 30-day mortality in the training and validation cohorts

\begin{tabular}{|c|c|c|c|c|c|c|}
\hline \multirow[t]{2}{*}{ Clinical characteristics } & \multicolumn{3}{|l|}{ Training cohort } & \multicolumn{3}{|l|}{ Validation cohort } \\
\hline & No. patients $(n=147)$ & 30-day mortality $(n=42)$ & $p$ value $^{a}$ & No. patients $(n=60)$ & 30-day mortality $(n=16)$ & $p$ value $^{a}$ \\
\hline Male (\%) & $75(51.0)$ & $20(47.6)$ & 0.715 & $36(60.0)$ & $9(56.3)$ & 0.771 \\
\hline Age > = 65 years old $(\%)$ & $68(46.3)$ & $21(50.0)$ & 0.587 & $22(36.7)$ & $6(37.5)$ & 1.000 \\
\hline LOS > = 30 days (\%) & $50(34.0)$ & $18(42.9)$ & 0.179 & $19(31.7)$ & $5(31.3)$ & 1.000 \\
\hline CVC > 7 days (\%) & 79 (53.7) & $24(57.1)$ & 0.715 & $35(58.3)$ & $12(75.0)$ & 0.146 \\
\hline TPN > 5 days (\%) & $54(36.7)$ & $16(38.1)$ & 0.851 & $21(35.0)$ & $7(43.8)$ & 0.541 \\
\hline Urinary catheter > 2 days (\%) & $41(27.9)$ & $13(31.0)$ & 0.685 & $21(35.0)$ & $8(50.0)$ & 0.220 \\
\hline Nasogastric tube > 3 days (\%) & $60(40.8)$ & $14(33.3)$ & 0.270 & $24(40.0)$ & $7(43.8)$ & 0.771 \\
\hline No prior surgery (\%) & $68(46.3)$ & 30 (71.4) & $<0.001$ & $41(68.3)$ & $14(87.5)$ & 0.066 \\
\hline Neutropenia (\%) & $9(6.1)$ & $3(7.1)$ & 0.715 & $3(5.0)$ & $2(12.5)$ & 0.171 \\
\hline Chemotherapy (\%) & $50(34.0)$ & $15(35.7)$ & 0.848 & $11(18.3)$ & $4(25.0)$ & 0.462 \\
\hline Metastasis (\%) & $50(34.0)$ & $28(66.7)$ & $<0.001$ & $24(40.0)$ & $9(56.3)$ & 0.145 \\
\hline ICU admitted > 3 days (\%) & $54(36.7)$ & $26(61.9)$ & $<0.001$ & $26(43.3)$ & $11(68.8)$ & 0.021 \\
\hline Previous antibiotics exposure (\%) & $104(70.7)$ & $31(73.8)$ & 0.691 & $41(68.3)$ & $10(62.5)$ & 0.550 \\
\hline Timely anti-fungal treatment & $101(68.7)$ & $31(73.8)$ & 0.437 & $42(70.0)$ & $14(87.5)$ & 0.112 \\
\hline Fluconazole prophylaxis & $11(7.5)$ & $5(11.9)$ & 0.295 & $9(15.0)$ & $5(31.3)$ & 0.048 \\
\hline No source control & $59(40.1)$ & $32(76.2)$ & $<0.001$ & $29(48.3)$ & $11(68.8)$ & 0.081 \\
\hline \multicolumn{7}{|l|}{ Site of infection } \\
\hline Bloodstream & $51(34.7)$ & $25(59.5)$ & $<0.001$ & $22(36.7)$ & $11(68.8)$ & 0.005 \\
\hline Abdominal cavity & $67(45.6)$ & $12(28.6)$ & 0.010 & $33(55.0)$ & $4(25.0)$ & 0.008 \\
\hline Thoracic cavity & $17(11.6)$ & $3(7.1)$ & 0.397 & $2(3.3)$ & 0 & 1.000 \\
\hline Pelvic cavity & $12(8.2)$ & $2(4.8)$ & 0.510 & $3(5.0)$ & $1(6.3)$ & 1.000 \\
\hline \multicolumn{7}{|l|}{ Candida species } \\
\hline Candida albicans & $72(49.0)$ & $18(42.9)$ & 0.367 & $28(46.7)$ & $4(25.0)$ & 0.077 \\
\hline Candida glabrata complex & $40(27.2)$ & $16(38.1)$ & 0.068 & $10(16.7)$ & $6(37.5)$ & 0.017 \\
\hline Candida tropicalis & $11(7.5)$ & $3(7.1)$ & 1.000 & $10(16.7)$ & $3(18.8)$ & 1.000 \\
\hline Candida parapsilosis complex & $9(6.1)$ & $3(7.1)$ & 0.715 & $6(10.0)$ & $2(12.5)$ & 0.653 \\
\hline Other Candida species ${ }^{\mathrm{b}}$ & $15(10.2)$ & $2(4.8)$ & 0.233 & $6(10.0)$ & $1(6.3)$ & 1.000 \\
\hline \multicolumn{7}{|l|}{ Tumor types } \\
\hline Gastrointestinal & $50(34.0)$ & $13(31.0)$ & 0.702 & $23(38.3)$ & $7(43.8)$ & 0.765 \\
\hline Hepatic-pancreatic & $57(38.8)$ & $19(45.2)$ & 0.351 & $22(36.7)$ & $4(25.0)$ & 0.367 \\
\hline Hematologic malignancy & $9(6.1)$ & $4(9.5)$ & 0.276 & $4(6.7)$ & $2(12.5)$ & 0.287 \\
\hline Others $^{c}$ & $31(21.1)$ & $6(14.3)$ & 0.265 & $11(18.3)$ & $3(18.8)$ & 1.000 \\
\hline \multicolumn{7}{|l|}{ Co-morbidities } \\
\hline Diabetes mellitus & $28(19.0)$ & $9(21.4)$ & 0.647 & $14(23.3)$ & $4(25.0)$ & 1.000 \\
\hline Live dysfunction & $30(20.4)$ & $9(21.4)$ & 0.825 & $14(23.3)$ & $4(25.0)$ & 1.000 \\
\hline Kidney dysfunction & $23(15.6)$ & $8(19.0)$ & 0.462 & $4(6.7)$ & $3(18.8)$ & 0.054 \\
\hline Respiratory dysfunction & $22(15.0)$ & $4(9.5)$ & 0.311 & $6(10.0)$ & $3(18.8)$ & 0.328 \\
\hline Cardiovascular dysfunction & $20(13.6)$ & $8(19.0)$ & 0.286 & $1(1.7)$ & $1(6.3)$ & 0.267 \\
\hline
\end{tabular}

LOS length of hospital stay, CVC central venous catheters, TPN total parenteral nutrition, ICU intensive care unit

aisher exact test were compared between patient alive vs deceased in 30-day

$\mathrm{b}$ In the training cohort: Candida lusitaniae $(n=7)$, Candida ciferrii $(n=3)$, Candida famata $(n=4)$ and Candida krusei ( $n=1)$; In the validation cohort: Candida lusitaniae $(n=2)$, Candida ciferrii $(n=2)$, Candida famata $(n=1)$ and Candida krusei $(n=1)$

c In the training cohort: lung cancer $(n=11)$, ovarian cancer $(n=7)$, renal cancer $(n=3)$, thymoma $(n=3)$, breast cancer $(n=2)$, giiomas $(n=1)$, rhabdomyosarcoma $(n=1)$, endometrial cancer $(n=1)$, liposarcoma $(n=1)$, nephroblastoma $(n=1)$; In the validation cohort: lung cancer $(n=2)$, ovarian cancer $(n=2)$, renal cancer $(n=2)$, cervical cancer $(n=2)$, nephroblastoma $(n=1)$, liposarcoma $(n=1)$, fibrosarcoma $(n=1)$

admitted $>3$ days, no prior surgery, metastasis and no source control. ICU admission as a predictor for death was consistent with other studies [7, 23, 24]. Previous articles reported that the crude mortality of ICU patients with invasive candidiasis reaches $42.8-58.6 \%[1,25]$. Source control refers to all physical actions taken to control a focus of infection, including reducing the burden or growth conditions favorable for microorganisms 
Table 2 Predictive model constructed by AIC in a Stepwise Algorithm in the training cohort

\begin{tabular}{llllll}
\hline Variables & OR & $\mathbf{9 5 \%} \mathbf{C l}$ & $\boldsymbol{p}$ value & AIC \\
\hline Model & Bloodstream infection & 4.097 & $1.478-12.131$ & 0.008 & 118.2 \\
& ICU admitted $>$ 3 days & 4.877 & $1.812-14.257$ & 0.002 & \\
No prior surgery & 3.466 & $1.316-9.777$ & 0.014 & \\
Metastasis & 4.685 & $1.787-12.942$ & 0.002 & \\
No source control & 4.826 & $1.830-13.528$ & 0.002 & \\
\hline
\end{tabular}

a Stepwise Algorithm, direction = "backward"

$O R$ odds ratio, $\mathrm{Cl}$ confidence interval, AIC Akaike's Information Criterion

[26]. Previous studies on abdominal candidiasis found that source control is a crucial determinant of survival, and the absence of adequate abdominal source control has been associated with mortality [26, 27]. In the current study, we also confirmed that no source control is a predictor for mortality due to invasive candidiasis.

Bloodstream infection as a predictor of 30-day death from invasive candidiasis has rarely been reported. Invasive candidiasis is commonly divided into bloodstream and deep-seated infections, such as intra-abdominal and intrathoracic abscesses, organ infections or osteomyelitis [5]. Compared to bloodstream infection (removing the intravenous catheter is the common method for source control, but it can only provide transient fungal burden reduction, and the source of infection is difficult to define most of the time), deep-seated infections in the current study occurred at the abdominal, thoracic and pelvic cavities. Source control, such as drainage (could continuously reduce the fungal burden), is easy to perform, which is important for a successful outcome [28]. This might partially explain why deep-seated infections had low mortality and bloodstream infections were associated with a poor prognosis in this study. A surveillance investigation also reported a low mortality of invasive candidiasis (30-day mortality rate of $12.3 \%)$, and the researchers considered it to be associated with a low ratio of candidemia and ICU stay [23].

As one of the predictors, metastasis has always been found accompanied by immunosuppression, which is associated with grim outcomes of infections due to Candidia spp. [29, 30]. On the other hand, previous study proved that Candida spp. could also accelerate the cancerous processes [31]. Thus, we speculated that the poor outcomes depend on the interaction of cancer metastasis and invasive candidiasis, which need to be further studied.

Interestingly, we found that patients who did not undergo surgery had a poor prognosis in invasive candidiasis. Previous studies also found that surgery was associated with a higher probability of survival $[11,32,33]$. To our knowledge, surgery is usually performed on patients without severe underlying diseases, especially among cancer patients. This might be the reason for the patients who were treated with surgery had favorable outcomes. However, one study reported that surgery was associated with high mortality of invasive candidiasis [24]. The discrepant findings might be associated with the types of surgery in the study population, as Orsetti E et al. pointed out that the mortality of candidemia varies among surgery types [34].

In general, hematologic malignancy and fluconazole prophylaxis were not associated with the outcome of invasive candidiasis in this study, which was inconsistent with some investigations for hematologic malignancy that related a poor outcome and fluconazole prophylaxis to a favorable outcome $[11,35]$. This might be partially explained by the limited number of cases (13 cases of hematologic malignancy and 20 cases of fluconazole prophylaxis) in our study, and the association needs further investigation. Protection against poor outcomes was also not related to timely antifungal treatment in the study, and we focused on 30-day crude mortality rather than mortality attributed to invasive candidiasis among cancer patients might be explained this. Death might mainly be due to cancer, and invasive candidiasis merely drives progression, which may have reduced the rate of successful therapy of timely antifungal treatment in the study population. A previous study also reported that antifungal treatments significantly reduced mortality for the first 7 days (most of the morality attributable to invasive candidiasis would be expected to occur within approximately 7 days) but showed no significant differences in 30-day mortality [36].

Among the whole population of this study, C. albicans was the predominant species, consistent with most recent reports [37, 38]. However, the C. glabrata complex ranked second, which was not highly consistent with other studies, with differences in population, region and study design; for example, some articles report that $C$. tropicalis is the dominant species in infections caused by non-albicans Candida [7]. Moreover, Candida species were not linked to 30-day crude mortality in the present study. Nonetheless, other studies found some relationships between Candida species and mortality. Viscoli C et al. observed that the C. glabrata complex was associated with the highest mortality rate compared with other Candida species [39], and Tedeschi S et al. reported that infection due to $C$. tropicalis was an independent risk factor for in-hospital mortality [40]. Hirano $\mathrm{R}$ et al. found that $C$. albicans was associated with a high mortality rate [33]. Khatib $\mathrm{R}$ et al. showed that the 30-day mortality rate was not different between $C$. albicans and the C. glabrata complex [41]. Combining these findings, we consider that the specificity of Candida speciesrelated mortality is associated with the study cohort rather than the species itself, i.e., the initial azole monotherapy was associated with worse outcome for cancer patient infected by fluconazole-susceptible dosedependent C. glabrata complex [42]. 


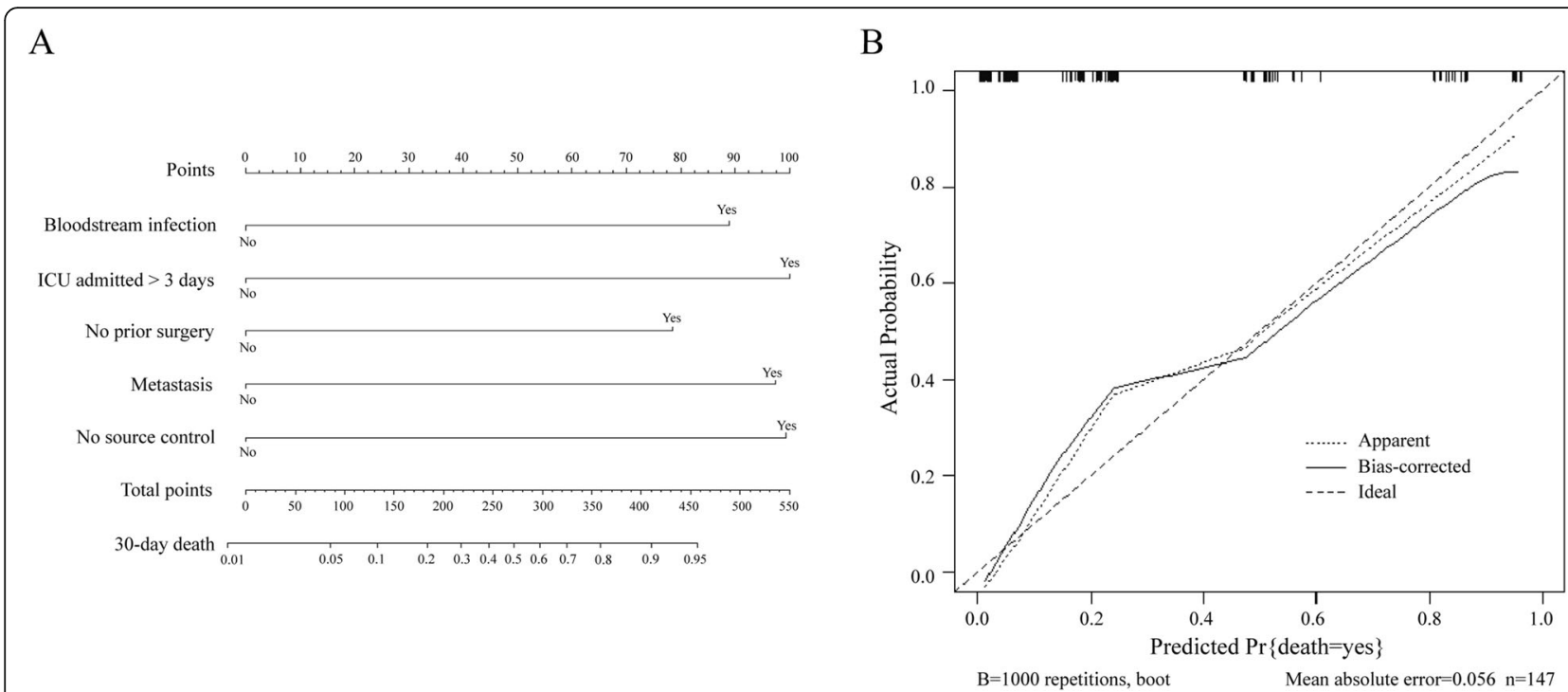

Fig. 2 Predictive nomogram and calibration plot. a. A predictive nomogram for predicting 30-day death of invasive candidiasis among cancer patients. To estimate the probability of 30-day death for a given patient, the following were carried out: patient values at each axis were marked, a straight line perpendicular to the point axis was drawn, and the points for all variables were summed. Then, we summed the total points and drew a vertical line from the "total points" row to obtain the probability of 30-day death. $\mathbf{b}$. A calibration plot of the predicted and observed probabilities of 30-day death of invasive candidiasis among cancer patients. The $x$-axis shows the predicted probability of 30-day death, and the $y$-axis shows the observed probability of 30-day death. The nomogram had a C-index of 0.895 and was well calibrated

\section{Conclusions}

In conclusion, the crude 30-day mortality of the whole cohort was $28.0 \%$, and variables including bloodstream infection, ICU admitted $>3$ days, no prior surgery, metastasis and no source control were predictors for mortality. Based on these predictors, we established a predictive nomogram that has good performance in prognosis prediction as well as clinical treatment strategies for invasive candidiasis among cancer patients. We believe that the predictive nomogram will benefit
A

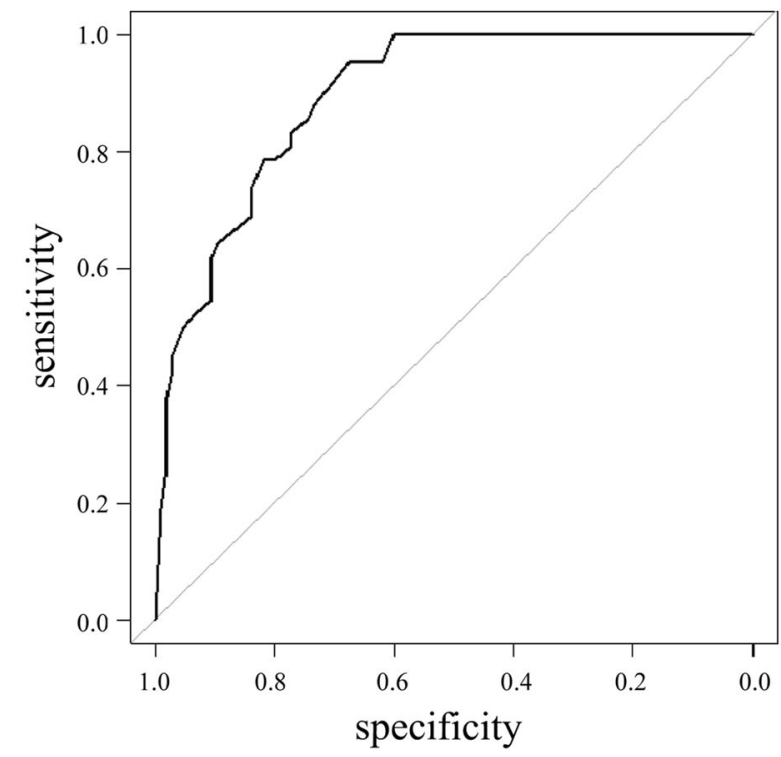

B

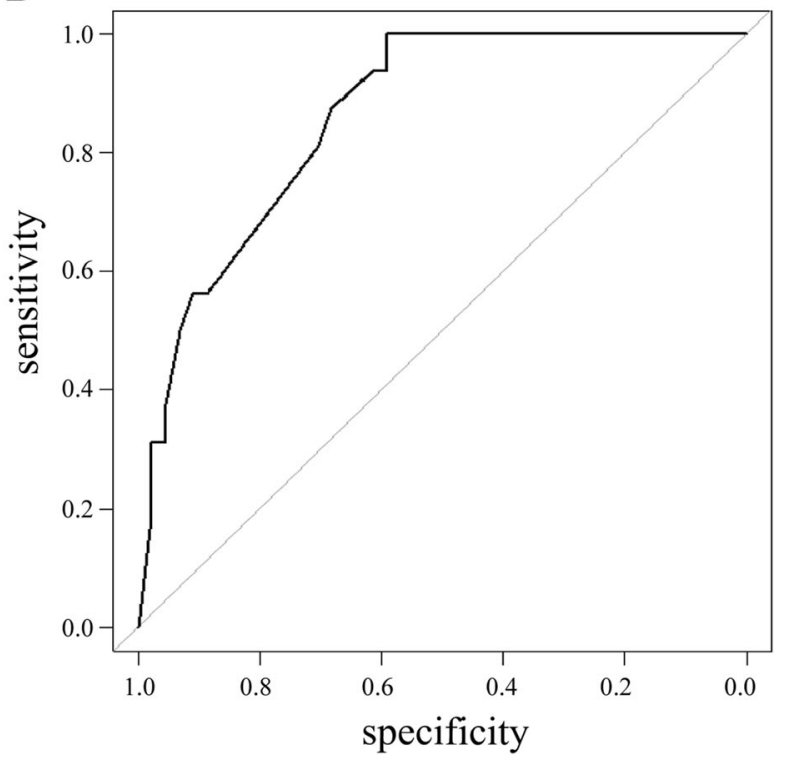

Fig. 3 ROC curve for the predictive nomogram of 30-day death among cancer patients with invasive candidiasis. a. ROC curve for the training cohort; the AUC was 0.895 (95\% confidence interval, 0.846-0.945). b. ROC curve for the validation cohort; the AUC was 0.862 ( $95 \%$ confidence interval, 0.770-0.955). Predictor variables are bloodstream infection, ICU admitted $>3$ days, no prior surgery, metastasis and no source control 
A

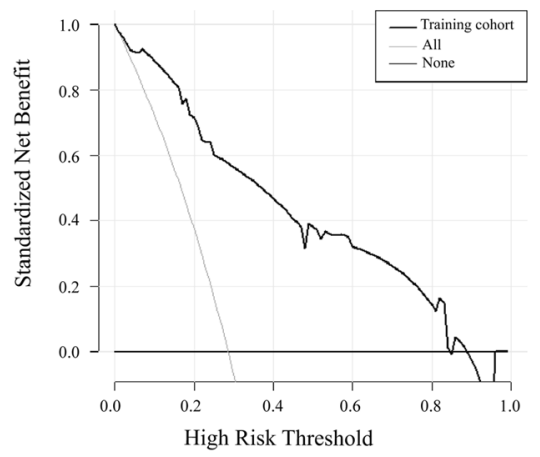

$\mathrm{C}$

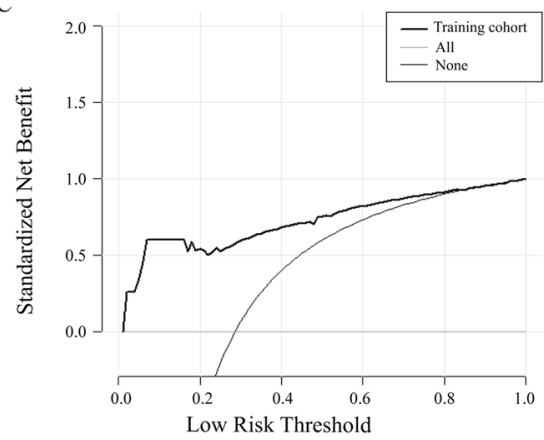

B

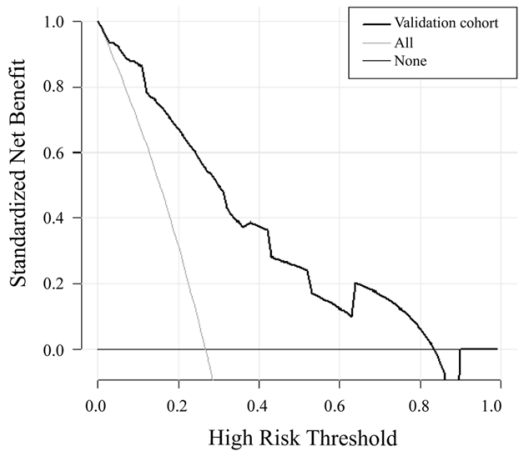

D

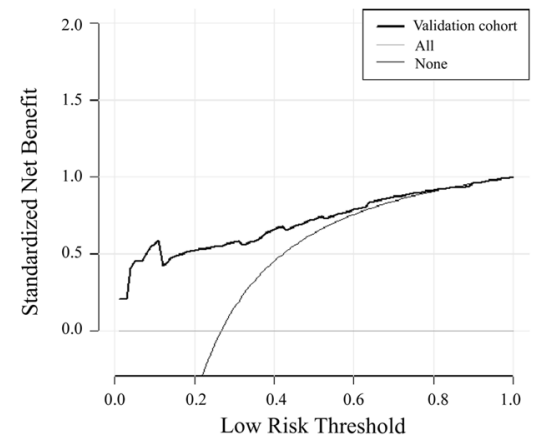

Fig. 4 Potential clinical impact of the predictive nomogram for the proposed intervention. The X-axis indicates that the threshold probability refers to the relative harm and benefit of the proposed intervention; the Y-axis indicates that the standardized net benefit. $\mathbf{a}$. Decision curve analysis for the training cohort. $\mathbf{b}$. Decision curve analysis for the validation cohort. c. Opt-out decision curve analysis for the training cohort. $\mathbf{d}$. Opt-out decision curve analysis for the validation cohort

outcome improvement. However, there are still several limitations in this study. This was a single-center investigation that did not use an external population to validate the predictive nomogram. In the future, a multicenter study with external validation should be performed, which may provide more relevant epidemiological information.

\section{Abbreviations}

AIC: Akaike's information criterion; AUC: The area under the receiver operating characteristic curve; CVC: Central venous catheter; Cl: Confidence interval; C-index: Concordance index; DCA: Decision curve analysis; ICU: Intensive care unit; LOS: Length of hospital stay; ROC: Receiver operating characteristic; TPN: Total parenteral nutrition

\section{Acknowledgments}

Not applicable.

\section{Authors' contributions}

Conceptualization and study design: DL, XL. Data collection: TL, QZ. Data analysis: $\mathrm{DL}, \mathrm{CB}, \mathrm{ZL}$. Writing: $\mathrm{DL}$. Reviewing and editing: $\mathrm{TL}, \mathrm{CB}, \mathrm{XL}$. All authors read and approved the manuscript.

\section{Funding}

This work was supported by grants from the National Natural Science Foundation of China (81802080 to Ding Li), which played a role in the design of the study. National Natural Science Foundation of China (81472681 and 818722361 to Xichuan Li), which had a role in the data collection. The grant from Natural Science Foundation of Tianjin (18JCQNJC80100 to Ding Li, 18JCYBJC28100 to Xichuan Li), which had a role in the data analysis. The grant from Tianjin Municipal Education Commission (2019KJ189 to Changsen Bai), which had a role in the manuscript writing, reviewing and editing.

\section{Availability of data and materials}

The datasets used and analyzed during the current study are available from the corresponding author on reasonable request.

\section{Ethics approval and consent to participate}

Ethics, consent and permissions: All procedures performed were in accordance with the ethical standards of the institutional research committee and with the 1964 Helsinki declaration and its later amendments or comparable ethical standards. This current study was approved by the Bioethics Committee of Tianjin Medical University Cancer Institute and Hospital. This article does not contain any studies with animals performed by any of the authors. Consent to participate in the study was obtained from all participants (written, participants under 16 years old written by their parents or legal guardians).

\section{Consent for publication}

Not applicable.

\section{Competing interests}

The authors declare that they have no competing interests.

\section{Author details}

${ }^{1}$ Department of Clinical Laboratory, Tianjin Medical University Cancer Institute and Hospital, National Clinical Research Center for Cancer, Key Laboratory of Cancer Prevention and Therapy, Tianjin's Clinical Research Center for Cancer, Huanhu West Road, Hexi District, Tianjin 300060, China. ${ }^{2}$ State Key Laboratory of Medicinal Chemical Biology, College of Pharmacy, Nankai University, Tianjin, China. ${ }^{3}$ Tianjin Key Laboratory of Animal and Plant Resistance, College of Life Sciences, Tianjin Normal University, Binshuixi Road, Tianjin 300387, Xiqing District, China. 
Received: 15 July 2020 Accepted: 7 January 2021

Published online: 15 January 2021

\section{References}

1. Bassetti M, Giacobbe DR, Vena A, Trucchi C, Ansaldi F, Antonelli M, et al. Incidence and outcome of invasive candidiasis in intensive care units (ICUs) in Europe: results of the EUCANDICU project. Crit Care. 2019;23:219.

2. Cleveland AA, Harrison LH, Farley MM, Hollick R, Stein B, Chiller TM, et al. Declining incidence of candidemia and the shifting epidemiology of Candida resistance in two US metropolitan areas, 2008-2013: results from population-based surveillance. PLoS One. 2015;10:e0120452.

3. Magill SS, O'Leary E, Janelle SJ, Thompson DL, Dumyati G, Nadle J, et al. Changes in prevalence of health care-associated infections in U.S. hospitals. N Engl J Med. 2018;379:1732-44.

4. Lortholary O, Renaudat C, Sitbon K, Madec Y, Denoeud-Ndam L, Wolff M, et al. Worrisome trends in incidence and mortality of candidemia in intensive care units (Paris area, 2002-2010). Intensive Care Med. 2014;40: 1303-12.

5. Pappas PG, Lionakis MS, Arendrup MC, Ostrosky-Zeichner L, Kullberg BJ. Invasive candidiasis. Nat Rev Dis Primers. 2018:4:18026.

6. Diekema DJ, Messer SA, Brueggemann AB, Coffman SL, Doern GV, Herwaldt $L A$, et al. Epidemiology of candidemia: 3-year results from the emerging infections and the epidemiology of lowa organisms study. J Clin Microbiol. 2002:40:1298-302

7. Lortholary O, Renaudat C, Sitbon K, Desnos-Ollivier M, Bretagne S, Dromer F, et al. The risk and clinical outcome of candidemia depending on underlying malignancy. Intensive Care Med. 2017;43:652-62.

8. Ortega-Loubon C, Cano-Hernández B, Poves-Alvarez R, Muñoz-Moreno MF, Román-García P, Balbás-Alvarez S, et al. The overlooked immune state in Candidemia: a risk factor for mortality. J Clin Med. 2019:8:1512.

9. Doi AM, Pignatari AC, Edmond MB, Marra AR, Camargo LF, Siqueira RA, et al. Epidemiology and microbiologic characterization of nosocomial Candidemia from a Brazilian National Surveillance Program. PLoS One. 2016; 11:e0146909.

10. León C, Ruiz-Santana S, Saavedra P, Galván B, Blanco A, Castro C, et al. Usefulness of the "Candida score" for discriminating between Candida colonization and invasive candidiasis in non-neutropenic critically ill patients: a prospective multicenter study. Crit Care Med. 2009;37:1624-33.

11. Keighley C, Chen SC, Marriott D, Pope A, Chapman B, Kennedy K, et al. Candidaemia and a risk predictive model for overall mortality: a prospective multicentre study. BMC Infect Dis. 2019;19:445.

12. Xia R, Wang D. Risk factors of invasive candidiasis in critical cancer patients after various gastrointestinal surgeries: a 4-year retrospective study. Medicine. 2019;98:e17704.

13. Wang H, Liu N, Yin M, Han H, Yue J, Zhang F, et al. The epidemiology, antifungal use and risk factors of death in elderly patients with candidemia: a multicentre retrospective study. BMC Infect Dis. 2014;14:609.

14. Ma CF, Li FQ, Shi LN, Hu YA, Wang Y, Huang M, et al. Surveillance study of species distribution, antifungal susceptibility and mortality of nosocomial candidemia in a tertiary care hospital in China. BMC Infect Dis. 2013;13:337.

15. Barchiesi F, Orsetti E, Gesuita R, Skrami E, Manso E. Candidemia study group. Epidemiology, clinical characteristics, and outcome of candidemia in a tertiary referral center in Italy from 2010 to 2014. Infection. 2016;44:205-13.

16. Balachandran VP, Gonen M, Smith JJ, DeMatteo RP. Nomograms in oncology: more than meets the eye. Lancet Oncol. 2015;16:e173-80.

17. Callegaro D, Miceli R, Mariani L, Raut CP, Gronchi A. Soft tissue sarcoma nomograms and their incorporation into practice. Cancer. 2017;123:2802-20.

18. lasonos A, Schrag D, Raj GV, Panageas KS. How to build and interpret a nomogram for cancer prognosis. J Clin Oncol. 2008;26:1364-70.

19. Vickers AJ, Elkin EB. Decision curve analysis: a novel method for evaluating prediction models. Med Decis Mak. 2006;26:565-74.

20. Vickers AJ, van Calster B, Steyerberg EW. A simple, step-by-step guide to interpreting decision curve analysis. Diagn Progn Res. 2019;3:18.

21. Vickers AJ, Van Calster B, Steyerberg EW. Net benefit approaches to the evaluation of prediction models, molecular markers, and diagnostic tests. BMJ. 2016;352:i6.

22. Kerr KF, Brown MD, Marsh $\mathrm{TL}$, Janes $\mathrm{H}$. Assessing the clinical impact of risk models for opting out of treatment. Med Decis Mak. 2019;39:86-90.

23. Zeng ZR, Tian G, Ding YH, Yang K, Liu JB, Deng J. Surveillance study of the prevalence, species distribution, antifungal susceptibility, risk factors and mortality of invasive candidiasis in a tertiary teaching hospital in Southwest China. BMC Infect Dis. 2019:19:939.

24. Leroy O, Bailly S, Gangneux JP, Mira JP, Devos P, Dupont H, et al. Systemic antifungal therapy for proven or suspected invasive candidiasis: the AmarCAND 2 study. Ann Intensive Care. 2016;6:2.

25. Montagna MT, Caggiano G, Lovero G, De Giglio O, Coretti C, Cuna T, et al. Epidemiology of invasive fungal infections in the intensive care unit: results of a multicenter Italian survey (AURORA project). Infection. 2013;41:645-53.

26. Lagunes L, Rey-Pérez A, Martín-Gómez MT, Vena A, de Egea V, Muñoz P, et al. Association between source control and mortality in 258 patients with intra-abdominal candidiasis: a retrospective multi-centric analysis comparing intensive care versus surgical wards in Spain. Eur J Clin Microbiol Infect Dis. 2017;36:95-104

27. Bassetti M, Righi E, Ansaldi F, Merelli M, Scarparo C, Antonelli M, et al. A multicenter multinational study of abdominal candidiasis: epidemiology, outcomes and predictors of mortality. Intensive Care Med. 2015;41:1601-10.

28. Kollef M, Micek S, Hampton N, Doherty JA, Kumar A. Septic shock attributed to Candida infection: importance of empiric therapy and source control. Clin Infect Dis. 2012;54:1739-46.

29. Ghrenassia E, Mokart D, Mayaux J, Demoule A, Rezine I, Kerhuel L, et al. Candidemia in critically ill immunocompromised patients: report of a retrospective multicenter cohort study. Ann Intensive Care. 2019:9:62.

30. Liu Y, Cao X. Immunosuppressive cells in tumor immune escape and metastasis. J Mol Med (Berl). 2016;94:509-22.

31. Ramirez-Garcia A, Rementeria A, Aguirre-Urizar JM, Moragues MD, Antoran A, Pellon A, et al. Candida albicans and cancer: can this yeast induce cancer development or progression? Crit Rev Microbiol. 2016;42:181-93.

32. Luzzati R, Merelli M, Ansaldi F, Rosin C, Azzini A, Cavinato S, et al. Nosocomial candidemia in patients admitted to medicine wards compared to other wards: a multicentre study. Infection. 2016;44:747-55.

33. Hirano R, Sakamoto Y, Kitazawa J, Yamamoto S, Kayaba H. Epidemiology, practice patterns, and prognostic factors for candidemia; and characteristics of fourteen patients with breakthrough Candida bloodstream infections: a single tertiary hospital experience in Japan. Infect Drug Resist. 2018;11:82133.

34. Orsetti E, Brescini L, Mazzanti S, Trave F, Morroni G, Masucci A, et al. Characterisation of candidemia in patients with recent surgery: a 7-year experience. Mycoses. 2019;62:1056-63.

35. Swoboda SM, Merz WG, Lipsetta PA. Candidemia: the impact of antifungal prophylaxis in a surgical intensive care unit. Surg Infect. 2003;4:345-54.

36. Poves-Alvarez R, Cano-Hernández B, Muñoz-Moreno MF, Balbás-Alvarez S, Román-García P, Gómez-Sánchez E, et al. Impact of empirical treatment with antifungal agents on survival of patients with candidemia. Rev Esp Quimioter. 2019:32:6-14.

37. Chapman B, Slavin M, Marriott D, Halliday C, Kidd S, Arthur I, et al. Changing epidemiology of candidaemia in Australia. J Antimicrob Chemother. 2017; 72:1103-8.

38. Wu JQ, Zhu LP, Ou XT, Xu B, Hu XP, Wang $X$, et al. Epidemiology and risk factors for non-Candida albicans candidemia in non-neutropenic patients at a Chinese teaching hospital. Med Mycol. 2011:49:552-5.

39. Viscoli C, Girmenia C, Marinus A, Collette L, Martino P, Vandercam B, et al. Candidemia in cancer patients: a prospective, multicenter surveillance study by the invasive fungal infection group (IFIG) of the European Organization for Research and Treatment of Cancer (EORTC). Clin Infect Dis. 1999;28: 1071-9.

40. Tedeschi S, Tumietto F, Giannella M, Bartoletti M, Cristini F, Cioni G, et al. Epidemiology and outcome of candidemia in internal medicine wards: a regional study in Italy. Eur J Intern Med. 2016;34:39-44.

41. Khatib R, Johnson LB, Fakih MG, Riederer K, Briski L. Current trends in candidemia and species distribution among adults: Candida glabrata surpasses $C$. albicans in diabetic patients and abdominal sources. Mycoses. 2016;59:781-6

42. Le A, Farmakiotis D, Tarrand JJ, Kontoyiannis DP. Initial treatment of Cancer patients with fluconazole-susceptible dose-dependent Candida glabrata Fungemia: better outcome with an Echinocandin or Polyene compared to an azole? Antimicrob Agents Chemother. 2017;61:e00631-17.

\section{Publisher's Note}

Springer Nature remains neutral with regard to jurisdictional claims in published maps and institutional affiliations. 